\title{
Erratum to: Collective Dynamics in the Vicsek and Vectorial Network Models Beyond Uniform Additive Noise
}

\author{
Violet Mwaffo $^{1}$ - Ross P. Anderson ${ }^{1}$. \\ Maurizio Porfiri ${ }^{1}$ \\ Published online: 1 February 2016 \\ (C) Springer Science+Business Media New York 2016
}

\section{Erratum to: J Nonlinear Sci (2015) 25:1053-1076 DOI 10.1007/s00332-015-9260-y}

The authors regret that due to a mistake in the preparation of the final figure set for the VM- $\omega$ model, Figs. 4, 5, 6, 7, and 12 do not correspond to the value of $r$ reported in the caption. The value of $r$ should be computed using $r=L \sqrt{(K-1) /((N-1) \pi)}$ to match the density of the VM- $\omega$ and the VNM- $\omega$ models.

Accordingly, these figures and their respective captions should be modified as follows. For $N=10$, the correct values of $r$ are $0.1881,0.3257,0.3761$, and 0.4976 for $K=2,4,5$, and 8, respectively; and for $N=5000$, the correct values of $r$ are 0.0080 , $0.0138,0.0160$, and $r=0.0211$ for $K=2,4,5$, and 8, respectively.

Consequently: i) in Fig. 4b, the order parameter reaches its steady state in more than 300 time steps as opposed to the originally plotted quantity - the values of the polarization after the initial transient are almost identical in the presence or in the absence of jumps; ii) in Fig. 5b, the color-bar minimum value is about 0.78 , rather than 0.82 as originally reported-no further differences are noted; and iii) Figs. 6b, d, $7 \mathrm{~b}$, and 12 should be replaced with those reported below.

The statements reported in the text are not affected by this mistake.

The online version of the original article can be found under doi:10.1007/s00332-015-9260-y.

Maurizio Porfiri

mporfiri@nyu.edu

1 Department of Mechanical and Aerospace Engineering, New York University Tandon School of Engineering (previously New York University Polytechnic School of Engineering), 6 MetroTech Center, Brooklyn, NY 11201, USA 


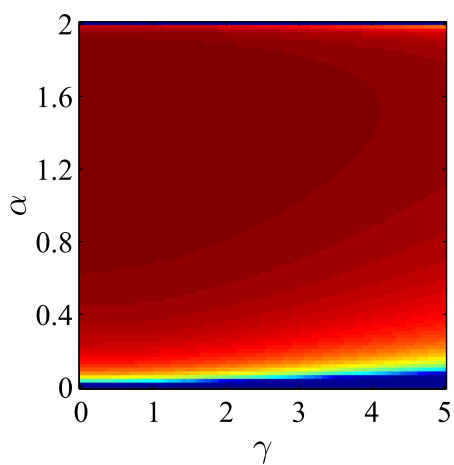

(b)
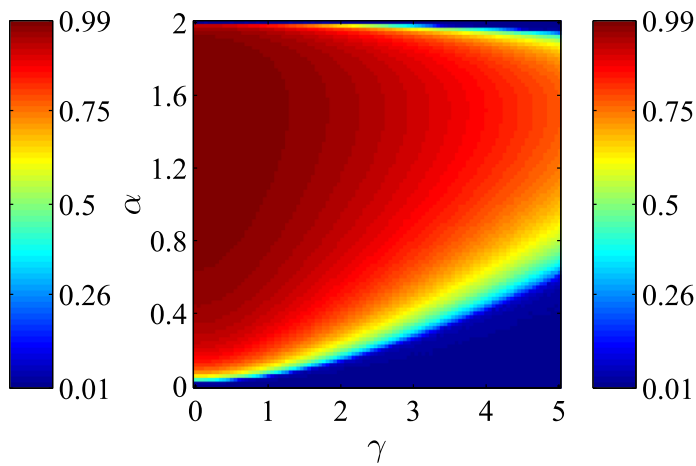

(d)

Fig. 6 Polarization as a function of the relaxation rate $\alpha$ and the jump amplitude $\gamma$ for $\mathbf{b} N=5000$ and $\lambda=0.1$ and $\mathbf{d} N=5000$ and $\lambda=1$, where $r=0.0080, \eta=0.1$, and $v=1$ (Color figure online)

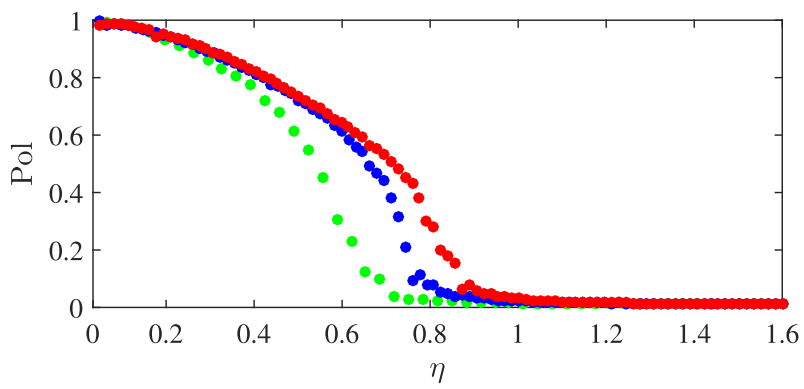

(b)

Fig. 7 (b) Polarization as a function of the noise scaling factor $\eta$ for $N=5000$, with varying radius of interaction ( $r=0.0080$ in green, $r=0.0160$ in blue, and $r=0.0211$ in red), with $\alpha=1.71, \lambda=0.1$, $\gamma=1$, and $v=0.01$ (Color figure online)

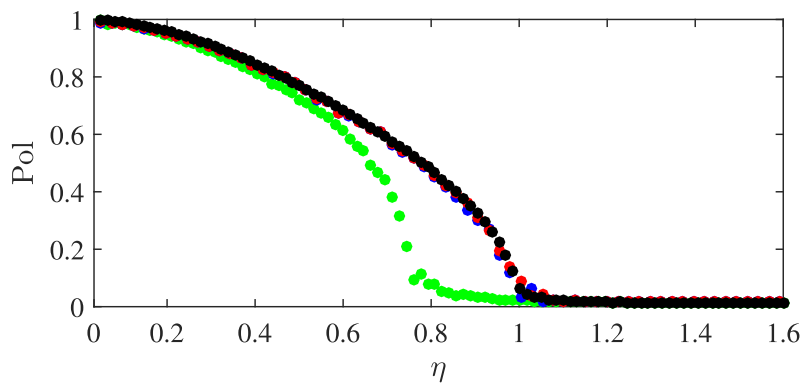

Fig. 12 The polarization of the VM- $\omega$ approaches that of the VNM- $\omega$ as the speed $v$ increases. Pictured are $v=0.01$ (green), $v=0.1$ (blue), $v=5$ (red), and the polarization of the VNM- $\omega$ is in black, with $\alpha=1.71, \lambda=0.1$, and $\gamma=1$. For the $\mathrm{VM}-\omega$, the radius of interaction is chosen as $r=0.0160$ so that the number of individuals within a circle of radius $r$ is on average the same as the number of individuals $K=5$ used in the VNM- $\omega$ (Color figure online) 